\title{
Formação para a prática de cuidado em oncologia nos cursos de ciências da saúde: revisão integrativa
}

\author{
Training for the practice of care in Oncology in health sciences courses: an integrative review \\ Formación para la práctica del cuidado en Oncología en los cursos de ciencias de la salud: una \\ revisión integradora
}

Recebido: 14/04/2021 | Revisado: 25/04/2021 | Aceito: 28/04/2021 | Publicado: 12/05/2021

\begin{abstract}
Clara Beatriz Teixeira Lima Cavalcante
ORCID: https://orcid.org/0000-0003-4798-7343 Universidade Federal do Estado do Rio de Janeiro, Brasil E-mail: clara.beatriz@edu.unirio.br

Vanessa de Almeida Ferreira Corrêa

ORCID: https://orcid.org/0000-0001-7121-4493 Universidade Federal do Estado do Rio de Janeiro, Brasil E-mail: vanessa.correa@unirio.br

Andressa Teoli Nunciaroni

ORCID: https://orcid.org/0000-0001-6469-592X Universidade Federal do Estado do Rio de Janeiro, Brasil E-mail: andressa.nunciaroni@unirio.br

Sônia Regina de Souza

ORCID: http://orcid.org/0000-0001-7981-0038 Universidade Federal do Estado do Rio de Janeiro, Brasil

E-mail: sonia.souza@unirio.br

Juliana Abreu de Vasconcellos

ORCID: https://orcid.org/0000-0001-7840-6551 Universidade Federal do Estado do Rio de Janeiro, Brasil E-mail: juliana.a.vasconcellos@gmail.com
\end{abstract}

\begin{abstract}
Resumo
Objetivo: Identificar, na literatura, as estratégias de ensino para a prática de cuidado em oncologia nos cursos de graduação da área da saúde. Metodologia: Trata-se de uma revisão integrativa nas bases de dados: LILACS; BDENF; MEDLINE; CINAHL e Web of Science, publicados no período de 2014 a 2020. Utilizou-se os descritores: Ciências da Saúde; Oncologia; Avaliação do Ensino e Universidades (Pesquisados no DeCS). Education, Graduate; Oncology and Universities (Pesquisados no MESH). 11 estudos compuseram o escopo da presente revisão. Resultados: Identificou-se 08 estratégias de ensino-aprendizagem, são elas: aulas teóricas e práticas; formação complementar através de cursos e disciplinas eletivas; uso de metodologias ativas através da discussão de casos, seminários e atividades educativas junto à população; e ligas acadêmicas. Quanto às estratégias de ensino voltadas aos princípios e diretrizes da Política Nacional para a Prevenção e Controle do Câncer na Rede de Atenção à Saúde, identificou-se as abordagens de: Promoção da saúde, prevenção e diagnóstico do câncer; tratamento e cuidados paliativos. Destaca-se que não se identificou estratégias de ensino voltadas às ações de detecção precoce, vigilância, monitoramento e avaliação. Conclusão: Salienta-se a necessidade de mais estudos na área, com o objetivo de promover a discussão de estratégias de ensino nos cursos de graduação da saúde que atendam aos princípios e diretrizes da Política Nacional para a Prevenção e Controle do Câncer na Rede de Atenção à Saúde das Pessoas com Doenças Crônicas no âmbito do Sistema Único de Saúde (SUS).
\end{abstract}

Palavras-chave: Ensino; Graduação; Oncologia; Ciências da saúde.

\begin{abstract}
Objective: To identify in the literature, the teaching strategies for the practice of care in oncology in undergraduate courses in the health area. Methodology: This is an integrative review in the databases: LILACS; BDENF; MEDLINE; CINAHL and Web of Science, published from 2014 to 2020. The descriptors used were: Health Sciences; Oncology; Evaluation of Teaching and Universities (Researched at DeCS). Education, Graduate; Oncology and Universities (Researched at MESH). 11 studies were included in the scope of the present review. Results: 08 teaching-learning strategies were identified, namely: theoretical and practical classes; complementary training through courses and electives; use of active methodologies through the discussion of cases, seminars and educational activities with the population; and academic leagues. As for teaching strategies aimed at the principles and guidelines of the National Policy for the Prevention and Control of Cancer in the Health Care Network, the following approaches were identified: Health promotion, cancer prevention and diagnosis; palliative care and treatment. It is noteworthy that teaching strategies aimed at early detection, surveillance, monitoring and evaluation actions were not identified. Conclusion: The
\end{abstract}


need for further studies in the area is emphasized, with the objective of promoting the discussion of teaching strategies in undergraduate health courses that meet the principles and guidelines of the National Policy for the Prevention and Control of Cancer in the Care Network to the Health of People with Chronic Diseases within the scope of the Unified Health System (SUS).

Keywords: Teaching; University graduate; Oncology; Health sciences.

\section{Resumen}

Objetivo: Identificar en la literatura, las estrategias de enseñanza para la práctica del cuidado en oncología en cursos de pregrado en el área de la salud. Metodología: Se trata de una revisión integradora en las bases de datos: LILACS; BDENF; MEDLINE; CINAHL y Web of Science, publicados de 2014 a 2020. Los descriptores utilizados fueron: Ciencias de la Salud; Oncología; Evaluación de Docencia y Universidades (Investigado en DeCS). Educación, Posgrado; Oncología y Universidades (Investigado en MESH). Se incluyeron 11 estudios en el alcance de la presente revisión. Resultados: Se identificaron 08 estrategias de enseñanza-aprendizaje que son: clases teóricas y prácticas; formación complementaria a través de cursos y optativas; uso de metodologías activas a través de la discusión de casos, seminarios y actividades educativas con la población; y ligas académicas. En cuanto a las estrategias de enseñanza orientadas a los principios y lineamientos de la Política Nacional para la Prevención y Control del Cáncer en la Red de Atención a la Salud, se identificaron los siguientes enfoques: Promoción de la salud, prevención y diagnóstico del cáncer; cuidados y tratamientos paliativos. Es de destacar que no se identificaron estrategias de enseñanza orientadas a acciones de detección temprana, vigilancia, seguimiento y evaluación. Conclusión: Se enfatiza la necesidad de profundizar los estudios en el área, con el objetivo de promover la discusión de estrategias docentes en los cursos de pregrado en salud que cumplan con los principios y lineamientos de la Política Nacional para la Prevención y Control del Cáncer en la Red de Atención a la Salud de las personas con enfermedades crónicas en el ámbito del Sistema Único de Salud (SUS).

Palabras clave: Enseñando; Graduado universitario; Oncología; Ciencias de la salud.

\section{Introdução}

O cuidado em oncologia abrange o conjunto de práticas e saberes voltados às diferentes profissões da área da saúde. Entende-se que, embora cada área tenha seu escopo de prática assistencial, a abordagem multidisciplinar é mais efetiva, quando comparada a sucessão de intervenções isoladas no cuidado ao paciente (Instituto Nacional De Câncer José Alencar Gomes Da Silva [INCA], 2020). O câncer é uma doença crônica não transmissível de grande impacto na saúde pública devido a sua alta morbimortalidade. A incidência e a mortalidade por câncer vêm aumentando no mundo, em parte pelo envelhecimento e pelo crescimento populacional, como também, pela mudança na distribuição e na prevalência dos fatores de risco de câncer, especialmente aos associados ao desenvolvimento socioeconômico (INCA, 2019).

A Portaria no 874 de 2013 instituiu, no Brasil a Política Nacional para a Prevenção e Controle do Câncer na Rede de Atenção à Saúde das Pessoas com Doenças Crônicas no âmbito do Sistema Único de Saúde (SUS); e contemplou as ações de: promoção, prevenção, detecção precoce, diagnóstico, tratamento e cuidados paliativos, como práticas multidisciplinares necessárias às equipes de saúde no SUS para a prevenção e controle da referida doença. Neste sentido, reitera-se a importância da prática de cuidado em oncologia, de forma multidisciplinar. Nos últimos anos, a oncologia evoluiu significativamente, com novas propostas terapêuticas e a inserção de tecnologias em saúde, sendo necessária a adoção de práticas multidisciplinares no cuidado em oncologia, nos diferentes pontos da atenção à saúde. Tal necessidade perpassa a formação de profissionais voltada às competências, habilidades e atitudes quanto à abordagem da prevenção e controle do câncer, sendo a formação em saúde e a educação permanente princípios da política em apreço (Portaria n. 874, 2013).

Cerca de um terço dos novos casos de câncer podem ser prevenidos através de ações de prevenção e promoção da saúde. A formação nos cursos de graduação na área da saúde devem contemplar à epidemiologia do câncer, aos fatores de risco oncológicos, o acompanhamento das pessoas com a doença e o impacto socioeconômico do câncer na população; associados às políticas públicas de saúde e educação, o que justifica a necessidade de conhecer como o assunto é abordado nos cursos de graduação na área da saúde, considerando que o câncer é um problema de saúde pública e em todo mundo, são esperados 28,4 milhões de novos casos de Câncer em 2040, um aumento de aproximadamente 47\% em relação a 2020 (Sung et al., 2021). 
A apreensão em redirecionar a formação em saúde, enquanto a aquisição de competências voltadas à prevenção, promoção, proteção e reabilitação da saúde; assim como vigilância em saúde, monitoramento e avaliação das ações em saúde, tanto em nível individual quanto coletivo, também é prevista nas Diretrizes Curriculares Nacionais dos Cursos de Graduação em Enfermagem, Medicina e Nutrição, sendo necessário a elaboração de currículos voltados ao enfrentamento das rápidas transformações da sociedade, do mercado de trabalho e das condições de exercício profissional (Resolução CNE/CES nº 3 , 2001).

Desta forma, o objetivo do presente estudo é identificar na literatura, as estratégias de ensino para a prática de cuidado em oncologia nos cursos de graduação da área da saúde.

\section{Metodologia}

Trata de uma revisão integrativa, desenvolvida em 6 etapas, conforme descrito por Souza et al. (2010). A primeira etapa se baseia na delimitação da questão norteadora: "Como as estratégias de ensino para a prática de cuidado em oncologia são abordadas nos cursos de graduação na área da saúde?”, através da estratégia PICo (P - População; I - Interesse; Co - Contexto), explicitada no Quadro 1.

Quadro 1. Estratégia PICo.

\begin{tabular}{|l|l|}
\hline PICo & Definição \\
\hline P & Cursos de graduação em ciências da saúde \\
\hline I & Estratégias de ensino para a prática de cuidado \\
\hline Co & Oncologia \\
\hline
\end{tabular}

Fonte: Dados da pesquisa (2021).

A segunda etapa constituiu a delimitação dos descritores através do DeCS (Descritores em Ciências da Saúde) para as bases de dados: Literatura Latino-Americana e do Caribe em Ciências da Saúde (LILACS); Base de dados de Enfermagem (BDENF); e Literatura Internacional em Ciências da Saúde (MEDLINE) e o MESH (Medical Subject Headings) para as bases de dados: CINAHL e Web of Science. Os descritores selecionados foram: Ciências da Saúde; Oncologia; Avaliação Educacional e Universidades (Pesquisados no DeCS). Education, Graduate; Oncology and Universities (Pesquisados no MESH). Os descritores foram pesquisados através de combinações utilizando o operador booleano "AND".

Os critérios de inclusão elencados foram: artigos completos nos idiomas inglês, português e espanhol e publicados no período de 2014 a 2020, os quais abordassem as estratégias de ensino para a prática de cuidado em oncologia durante a graduação nos cursos de ciências da saúde. Os critérios de exclusão relacionaram-se aos estudos que não abordassem o tema proposto, estudos de revisão integrativa e estudos voltados às especializações de residência, mestrado ou doutorado. Utilizou-se o software Mendeley® para gerenciamento dos artigos selecionados.

$\mathrm{Na}$ terceira etapa, realizou-se leitura dos títulos e resumos dos artigos previamente pesquisados, com seleção dos artigos condizentes com a questão norteadora. Excluiu-se, as duplicatas e estudos que não atendem ao objetivo da pesquisa. A quarta etapa se pautou na leitura na íntegra dos artigos selecionados, com o objetivo de analisar os critérios de inclusão e exclusão, e selecionar os artigos relevantes para a pesquisa. Ao final desta etapa, 11 estudos compuseram o escopo da presente revisão. Apresenta-se o fluxograma de buscas na Figura 1. 
Em seguida, na quinta etapa, realizou-se a tabulação dos dados, nos artigos selecionados, através da apresentação das estratégias de ensino em oncologia nos cursos de graduação em saúde; e as abordagens voltadas aos princípios e diretrizes da Política Nacional para a Prevenção e Controle do Câncer na Rede de Atenção à Saúde (RAS). Finalmente, na sexta etapa, foi realizada a apresentação da revisão.

Figura 1. Fluxograma de buscas de artigos no período de 2014 a 2020.

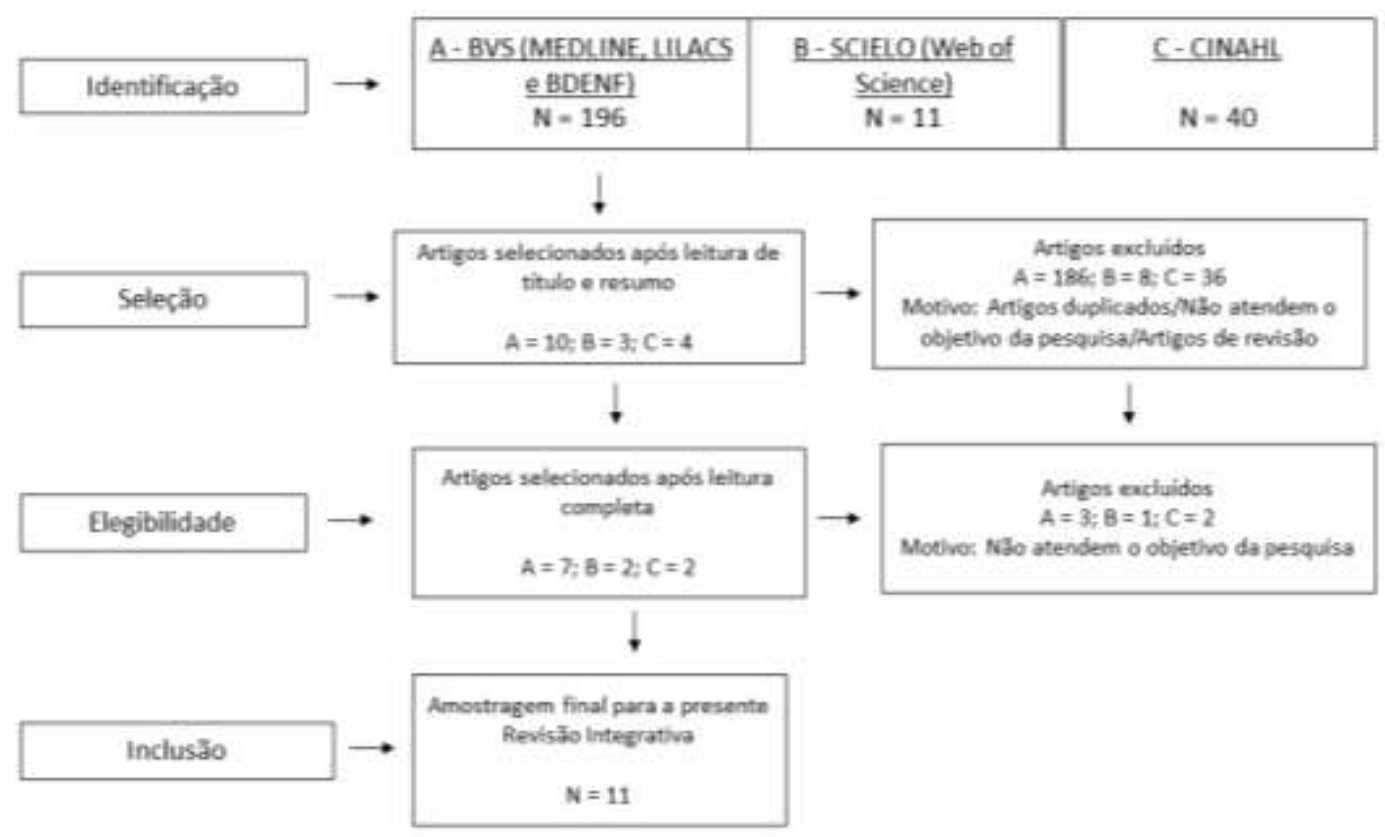

Fonte: Dados da pesquisa (2021).

\section{Resultados e Discussão}

Para a identificação dos artigos selecionados, construiu-se o Quadro 2: Identificação dos artigos por Autor/ano e Título dos artigos, Nível de Evidência de Oxford, local do estudo, objetivo e conclusão. Para a avaliação quanto ao nível de evidência dos estudos, utilizou-se a classificação da Oxford Centre for Evidence-based Medicine [CEBM] (2009). 
Quadro 2. Identificação do artigo por Autor/ano e Título dos artigos, Nível de Evidência de Oxford, local do estudo, objetivo e conclusão (2014 a 2020).

\begin{tabular}{|c|c|c|c|c|}
\hline $\begin{array}{l}\text { Autor/ano e Título dos } \\
\text { artigos }\end{array}$ & $\begin{array}{l}\text { Nível de } \\
\text { Evidência }\end{array}$ & Local do estudo & Objetivo & Conclusão \\
\hline $\begin{array}{l}\text { Mitchell, \& Laing } \\
\text { (2019). Revision of an } \\
\text { undergraduate nursing } \\
\text { oncology course using } \\
\text { the Taylor Curriculum } \\
\text { Review Process. }\end{array}$ & 3B & Canadá & $\begin{array}{l}\text { Revisar as estratégias de } \\
\text { ensino de um curso sobre } \\
\text { oncologia para graduandos } \\
\text { de enfermagem. }\end{array}$ & $\begin{array}{l}\text { É preciso manter constante } \\
\text { revisão do ensino de oncologia } \\
\text { na graduação de modo a } \\
\text { atualizar as estratégias e } \\
\text { temáticas abordadas. }\end{array}$ \\
\hline $\begin{array}{l}\text { Arenas, Sabater, Biete, } \\
\text { Lara \& Calvo (2018). } \\
\text { Radiation Oncology } \\
\text { Teaching Programmes as } \\
\text { Part of the } \\
\text { Undergraduate Degree in } \\
\text { Medicine in Spanish } \\
\text { Universities: the Need } \\
\text { for an Update of the } \\
\text { Contents and Structure. }\end{array}$ & $2 \mathrm{C}$ & Espanha & $\begin{array}{l}\text { Desenvolver um panorama } \\
\text { da situação do ensino de RO } \\
\text { (Radiation Oncology) na } \\
\text { educação universitária de } \\
\text { graduação na Espanha. }\end{array}$ & $\begin{array}{l}\text { O ensino da RO em } 40 \\
\text { universidades espanholas } \\
\text { apresenta carga horária e } \\
\text { estratégias de ensino } \\
\text { diversificadas. }\end{array}$ \\
\hline $\begin{array}{l}\text { Fox (2020). Why our } \\
\text { undergraduate nursing } \\
\text { programs need oncology } \\
\text { content: Reflections of a } \\
\text { nursing instructor. }\end{array}$ & 4 & Canadá & $\begin{array}{l}\text { Refletir sobre a importância } \\
\text { do ensino da oncologia na } \\
\text { graduação de enfermeiros }\end{array}$ & $\begin{array}{l}\text { A implementação de conteúdo } \\
\text { oncológico tem o potencial de } \\
\text { aumentar a competência de } \\
\text { nossos futuros enfermeiros, } \\
\text { expandir o interesse nesta área } \\
\text { de enfermagem e melhorar os } \\
\text { resultados dos pacientes. }\end{array}$ \\
\hline $\begin{array}{l}\text { Peñas-Felizzola, Parra- } \\
\text { Esquivel, \& Gómez- } \\
\text { Galindo. (2018). Terapia } \\
\text { ocupacional en } \\
\text { oncología: experiencias } \\
\text { en prácticas académicas } \\
\text { y revisión de literatura. }\end{array}$ & $3 \mathrm{~A}$ & Colômbia & $\begin{array}{l}\text { Consultar sobre a formação } \\
\text { de terapeutas ocupacionais } \\
\text { em oncologia em um } \\
\text { programa universitário. }\end{array}$ & $\begin{array}{l}\text { Menos de um quarto dos } \\
\text { estudantes respondeu ter } \\
\text { recebido ao longo do currículo } \\
\text { formação teórica sobre o } \\
\text { diagnóstico, gestão clínica ou } \\
\text { implicações da patologia e } \\
\text { sobre a intervenção do } \\
\text { terapeuta ocupacional com } \\
\text { esta população. }\end{array}$ \\
\hline
\end{tabular}




\begin{tabular}{|c|c|c|c|c|}
\hline $\begin{array}{l}\text { Guimarães, Silva, Santo, } \\
\text { Moraes \& Pacheco. } \\
\text { (2017). Cuidado paliativo } \\
\text { em oncologia pediátrica } \\
\text { na formação do } \\
\text { enfermeiro. }\end{array}$ & $2 \mathrm{C}$ & $\begin{array}{l}\text { Rio de Janeiro } \\
\text { (BR) }\end{array}$ & $\begin{array}{l}\text { Identificar e descrever a } \\
\text { visão dos acadêmicos de } \\
\text { enfermagem sobre os } \\
\text { cuidados paliativos em } \\
\text { oncologia pediátrica durante } \\
\text { a graduação. }\end{array}$ & $\begin{array}{l}\text { A importância da inserção do } \\
\text { tema cuidados paliativos em } \\
\text { oncologia pediátrica na } \\
\text { graduação em Enfermagem é } \\
\text { reconhecida por muitos } \\
\text { acadêmicos. }\end{array}$ \\
\hline $\begin{array}{l}\text { Rossato, Panobianco \& } \\
\text { Scorsolini-Comin. (2020) } \\
\text { Grupo operativo com } \\
\text { estudantes de } \\
\text { enfermagem: vivência } \\
\text { em uma Liga Acadêmica } \\
\text { de Oncologia. }\end{array}$ & 4 & $\begin{array}{l}\text { São Paulo } \\
\text { (BR) }\end{array}$ & $\begin{array}{l}\text { Descrever uma atividade } \\
\text { grupal desenvolvida em uma } \\
\text { Liga Acadêmica de } \\
\text { Prevenção ao Câncer }\end{array}$ & $\begin{array}{l}\text { Destaca-se a importância da } \\
\text { temática ser trabalhada nos } \\
\text { contextos de formação de } \\
\text { Enfermagem, a fim de que os } \\
\text { sujeitos adquiram repertório } \\
\text { teórico e técnico para lidar nas } \\
\text { situações de intervenção na } \\
\text { oncologia. }\end{array}$ \\
\hline $\begin{array}{l}\text { Aragão, Uchoa \& } \\
\text { Germano (2016). Ações } \\
\text { na sala de espera de } \\
\text { serviço de oncologia: } \\
\text { relato de experiência. }\end{array}$ & 4 & $\begin{array}{l}\text { Fortaleza } \\
(\mathrm{BR})\end{array}$ & $\begin{array}{l}\text { Relatar a experiência do } \\
\text { Projeto de Extensão Sala de } \\
\text { Espera, discutindo sua } \\
\text { importância no processo de } \\
\text { formação acadêmica na } \\
\text { Graduação em Psicologia. }\end{array}$ & $\begin{array}{l}\text { Ao entrar em contato com o } \\
\text { paciente e familiares, permite- } \\
\text { se a criação de estratégias de } \\
\text { educação em saúde e interação } \\
\text { com a realidade da pessoa com } \\
\text { câncer, sendo extremamente } \\
\text { importante pra formação do } \\
\text { profissional. }\end{array}$ \\
\hline $\begin{array}{l}\text { Freiser et al. (2016). } \\
\text { Educational Value of a } \\
\text { Medical Student-Led } \\
\text { Head and Neck Cancer } \\
\text { Screening Event. }\end{array}$ & $2 \mathrm{~B}$ & $\begin{array}{l}\text { Miami } \\
\text { (EUA) }\end{array}$ & $\begin{array}{l}\text { Avaliar a melhoria do } \\
\text { conhecimento do estudante } \\
\text { de medicina sobre o câncer } \\
\text { de cabeça e pescoço (HNC) } \\
\text { por meio da participação em } \\
\text { feiras de triagem do HNC } \\
\text { dirigidas por estudantes }\end{array}$ & $\begin{array}{l}\text { O conhecimento básico sobre } \\
\text { HNC continua baixo entre os } \\
\text { estudantes de medicina. É } \\
\text { necessário que os alunos de } \\
\text { todas as especialidades } \\
\text { recebam educação adicional } \\
\text { sobre HNC. }\end{array}$ \\
\hline $\begin{array}{l}\text { Vieira, Lopes, Sarri, } \\
\text { Benedetti \& Oliveira, } \\
\text { (2017). Oncology E- } \\
\text { Learning for } \\
\text { Undergraduate. A } \\
\text { Prospective Randomized } \\
\text { Controlled Trial. }\end{array}$ & 1B & $\begin{array}{l}\text { São Paulo } \\
\text { (BR) }\end{array}$ & $\begin{array}{l}\text { Avaliar o nível de retenção } \\
\text { de informações em } \\
\text { oncologia de alunos de } \\
\text { graduação em fisioterapia. }\end{array}$ & $\begin{array}{l}\text { As modalidades de } \text {-learning } \\
\text { são uma forma complementar } \\
\text { de aprimorar o conhecimento } \\
\text { em oncologia para alunos de } \\
\text { graduação. }\end{array}$ \\
\hline
\end{tabular}




\begin{tabular}{|c|c|c|c|c|}
\hline $\begin{array}{l}\text { Hokama, Hokama \& } \\
\text { Batista (2018). Caso } \\
\text { Motivador como } \\
\text { Estratégia } \\
\text { Problematizadora e } \\
\text { Integradora no Ensino } \\
\text { Médico em um Curso de } \\
\text { Oncologia. }\end{array}$ & 4 & $\begin{array}{l}\text { São Paulo } \\
\text { (BR) }\end{array}$ & $\begin{array}{l}\text { Relatar nossa experiência na } \\
\text { construção, aplicação e } \\
\text { avaliação de dois casos } \\
\text { motivadores voltados aos } \\
\text { discentes do quarto ano do } \\
\text { curso teórico de Oncologia } \\
\text { da Faculdade de Medicina de } \\
\text { Botucatu (Unesp). }\end{array}$ & $\begin{array}{l}\text { A partir da aplicação do Caso } \\
\text { Motivador, os alunos tiveram } \\
\text { a oportunidade de debater e } \\
\text { sintetizar em grupos os } \\
\text { diversos assuntos lecionados } \\
\text { sob um eixo norteador, no } \\
\text { caso, o Prognóstico na } \\
\text { Oncologia. }\end{array}$ \\
\hline $\begin{array}{l}\text { McKillip et al. (2019). } \\
\text { Implementation of a } \\
\text { Novel Medical School } \\
\text { Multidisciplinary and } \\
\text { Interprofessional } \\
\text { Oncology Curriculum: a } \\
\text { Mixed Method Study. }\end{array}$ & $2 \mathrm{~B}$ & $\begin{array}{l}\text { Chicago } \\
\text { (EUA) }\end{array}$ & $\begin{array}{l}\text { Melhorar a compreensão do } \\
\text { estudante de medicina do } \\
\text { primeiro ano do continuum } \\
\text { do tratamento do câncer, da } \\
\text { pesquisa oncológica e da } \\
\text { importância da medicina } \\
\text { interprofissional e } \\
\text { multidisciplinar. }\end{array}$ & $\begin{array}{l}\text { Ao oferecer um curso eletivo } \\
\text { de verão em oncologia, pôde- } \\
\text { se expandir com sucesso a } \\
\text { compreensão do tratamento e } \\
\text { pesquisa do câncer entre os } \\
\text { alunos de medicina do } \\
\text { primeiro ano. }\end{array}$ \\
\hline
\end{tabular}

Fonte: Dados da pesquisa (2021).

A extração dos dados dos artigos é exposta no Quadro 3, contendo as seguintes variáveis: estratégia de ensino, curso de graduação em saúde e abordagem voltada aos princípios e diretrizes da Política Nacional para a Prevenção e Controle do Câncer na RAS.

Quadro 3. Caracterização dos artigos por nome, população de estudo, estratégia de ensino apresentada, curso da saúde abordado e temática em oncologia discutida.

\begin{tabular}{|c|c|c|c|}
\hline Autor/Ano de publicação & Estratégia de ensino & Curso de graduação em saúde & $\begin{array}{l}\text { Abordagem voltada aos } \\
\text { princípios e diretrizes da } \\
\text { Política Nacional para a } \\
\text { Prevenção e Controle do } \\
\text { Câncer na RAS }\end{array}$ \\
\hline Mitchell, \& Laing (2019). & $\begin{array}{l}\text { Curso de oncologia } \\
\text {-Aulas teóricas } \\
\text { Discussão de casos } \\
\text { Seminários }\end{array}$ & Enfermagem & $\begin{array}{l}\text { Diagnóstico } \\
\text { Tratamento }\end{array}$ \\
\hline $\begin{array}{l}\text { Arenas, Sabater, Biete, } \\
\text { Lara \& Calvo (2018). }\end{array}$ & $\begin{array}{l}\text { Aulas Teóricas } \\
\text { Aulas práticas }\end{array}$ & Medicina & Tratamento \\
\hline Fox $(2020)$ & $\begin{array}{l}\text { Aulas teóricas } \\
\text { Aulas práticas }\end{array}$ & Enfermagem & Promoção (Educação em saúde) \\
\hline
\end{tabular}




\begin{tabular}{|c|c|c|c|}
\hline $\begin{array}{l}\text { Peñas-Felizzola, } \quad \text { Parra- } \\
\text { Esquivel, \& } \quad \text { Gómez- } \\
\text { Galindo. (2018). }\end{array}$ & Aulas teóricas & Terapia Ocupacional & Diagnóstico \\
\hline $\begin{array}{l}\text { Guimarães, Silva, Santo, } \\
\text { Moraes \& Pacheco. (2017) }\end{array}$ & $\begin{array}{l}\text { Aulas teóricas } \\
\text { Disciplina eletiva } \\
\text { sobre oncologia } \\
\text { Seminários }\end{array}$ & Enfermagem & Cuidados Paliativos \\
\hline $\begin{array}{l}\text { Rossato, Panobianco \& } \\
\text { Scorsolini-Comin. (2020) }\end{array}$ & Liga Acadêmica & Enfermagem & Promoção (Educação em saúde) \\
\hline $\begin{array}{l}\text { Aragão, Uchoa \& Germano } \\
\text { (2016). }\end{array}$ & $\begin{array}{l}\text { Atividades educativas } \\
\text { à população }\end{array}$ & Psicologia & $\begin{array}{l}\text { Diagnóstico } \\
\text { Promoção (Educação em saúde) }\end{array}$ \\
\hline Freiser et al. (2016). & $\begin{array}{l}\text { Atividades educativas } \\
\text { à população }\end{array}$ & Medicina & $\begin{array}{l}\text { Prevenção } \\
\text { Diagnóstico } \\
\text { Promoção (Educação em saúde) }\end{array}$ \\
\hline $\begin{array}{l}\text { Vieira, Lopes, Sarri, } \\
\text { Benedetti \& Oliveira, } \\
\text { (2017). }\end{array}$ & $\begin{array}{l}\text { Curso de Oncologia } \\
\text { Aulas teóricas }\end{array}$ & Fisioterapia & Cuidados Paliativos \\
\hline $\begin{array}{l}\text { Hokama, Hokama \& } \\
\text { Batista (2018). }\end{array}$ & $\begin{array}{l}\text { Discussão de caso } \\
\text { Seminários } \\
\text { Aulas teóricas e } \\
\text { práticas }\end{array}$ & Medicina & $\begin{array}{l}\text { Diagnóstico } \\
\text { Tratamento }\end{array}$ \\
\hline McKillip et al. (2019). & $\begin{array}{l}\text { Curso eletivo de } \\
\text { oncologia } \\
\text { Aulas teóricas }\end{array}$ & $\begin{array}{c}\text { Diversos cursos de Ciências } \\
\text { Biológicas }\end{array}$ & $\begin{array}{l}\text { Diagnóstico } \\
\text { Tratamento } \\
\text { Cuidados Paliativos }\end{array}$ \\
\hline
\end{tabular}

Fonte: Dados da pesquisa (2021).

Assim, para a melhor compreensão do tema e responder à questão norteadora desta pesquisa, a análise deste estudo apresenta as categorias: Estratégias de ensino em oncologia nos cursos de graduação em saúde; e as Abordagens voltadas aos princípios e diretrizes da Política Nacional de Prevenção e Controle do Câncer na RAS identificadas nas estratégias de ensino, a partir dos artigos científicos selecionados.

Categoria 1. Estratégias de ensino em oncologia nos cursos de graduação em saúde

Nas estratégias de ensino desenvolvidas, a partir da leitura dos artigos, identificou-se 08 estratégias de ensinoaprendizagem voltadas à oncologia, são elas: aulas teóricas e práticas; formação complementar através de cursos e disciplinas 
eletivas; uso de metodologias ativas através da discussão de casos, seminários e atividades educativas junto à população; e sensibilização de discentes quanto à temática do câncer, fomentado por liga acadêmica.

Quanto às aulas teóricas e práticas ministradas junto aos discentes de cursos de graduação, encontrou-se 08 (72,7\%) estudos que mencionam aulas teóricas no ensino da oncologia (Mitchell \& Laing, 2019; Arenas et al., 2018; Fox, 2020; PeñasFelizzola et al., 2017; Guimarães, et al., 2017; Vieira et al., 2016; Hokama et al, 2018; Mckillip et al., 2017) e 03 (27,2\%) artigos que referem o desenvolvimento de aulas práticas (Arenas et al., 2018; Fox, 2020; Hokama et al., 2018).

A aula teórica, ou aula expositiva dialogada, é uma das estratégias de ensino mais utilizadas e conhecidas pelos docentes. Baseia-se na exposição oral do conteúdo pelo docente, e pode contar com maior ou menor participação dos estudantes, dependendo da proposta e objetivos de ensino (Caveião et al., 2018). Destaca-se, a necessidade de instigar a participação discente no seu aprendizado e a integração de outras estratégias de ensino associadas à aula teórica, de modo que as características do ensino tradicional voltadas à centralização e autonomia do professor; reprodução do conhecimento e da avaliação possam ser superadas (Gonçalves et al., 2020).

O estudo de Mitchell e Laing (2019) traz a aula teórica como estratégia de ensino em um curso de oncologia, com aulas de 3 horas semanais, aliada a outras estratégias educativas. $O$ autor destaca que, o currículo do Curso de Enfermagem, conta com aulas expositivas para o ensino de diversos assuntos relativos ao câncer. Entende-se que, apesar da dinâmica de aula expositiva ser a mais comum, nas situações em que não há a participação do estudante, e o docente não o estimula, esta passa a ser somente uma metodologia tradicional, não havendo pensamento crítico (Caveião et al., 2018).

Arenas et al. (2018) apresenta em seu artigo, a integração do ensino teórico em oncologia com o ensino prático, em um curso de graduação em medicina. Ressalta-se que, as Diretrizes Curriculares Nacionais (DCN) indicam a necessidade de inserção de atividades teóricas e práticas ocorrerem desde o início do curso, de forma integrada e interdisciplinar (Costa et al., 2018). O estudo de Peñas-Felizzola et al., (2017) também realiza o diálogo entre a teoria e a prática, ao analisar se a formação em oncologia dos discentes em um curso de graduação em terapia ocupacional contém características práticas, para além da formação teórica. O estudo demonstrou número estatístico pequeno de participantes que relataram receber a formação teórico-prática em oncologia durante a sua graduação, o que demonstra uma lacuna de conhecimento pertinente para a presente discussão.

O estudo de Guimarães et al. (2017) cita o conteúdo teórico em oncologia através de uma aula disponibilizada no currículo obrigatório do curso de graduação em enfermagem. Entretanto, destaca-se que a aula ocorreu durante uma disciplina não relativa à oncologia. Os autores ainda destacam que, embora a oncologia não seja abordada através de uma disciplina própria, os alunos demonstram interesses em disciplinas obrigatórias, optativas e até cursos de capacitação que abordam o campo em apreço. Neste sentido, reitera-se a necessidade de abranger esse conteúdo durante a grade curricular obrigatória nos cursos de graduação de ciências da saúde.

É evidenciado, através da literatura, a existência de cursos de graduação que não possuem disciplinas de oncologia no currículo generalista (Lins \& Souza, 2018). Algumas instituições de ensino excluíram as referidas disciplinas, por entenderem a especificidade da oncologia referente ao ensino em cursos de pós-graduação lato sensu (Especialização), estando a oncologia entre elas (Calil \& Prado, 2010). Mckillip et al. (2017), ao apresentar a discussão sobre o ensino da oncologia, refere aulas teóricas ministradas por profissionais da área, pesquisadores e cientistas. É interessante ressaltar tal estratégia, visto que, a experiência da prática, se mostra essencial no ensino da oncologia. Para além do conteúdo teórico, o cotidiano do profissional inserido no cuidado traz uma abordagem importante para a formação do discente, a partir do cotidiano do serviço em saúde.

No que tange a inserção nos cenários de prática, além de possibilitar a correlação do que foi aprendido na teoria, é evidenciado que facilita a humanização do cuidado, visto que promove a interação entre o discente e paciente (C. Silva et al., 2019). A vivência da prática possibilita reflexões, estimula o desenvolvimento da empatia e a vontade de continuar a formação 
profissional de forma humanitária (Nalon et al., 2019). Se faz necessário, então, o contato com a prática o mais cedo possível, com a finalidade de aprimorar o ensino e incentivar a assistência humanizada (Nalon et al., 2019).

Outra estratégia de ensino identificada durante a análise dos artigos relaciona-se ao ensino da oncologia como externo à matriz curricular obrigatória, sendo identificado como "Formação Complementar", a qual compreende as seguintes estratégias mencionadas nos artigos: curso de oncologia e disciplina eletiva. Ainda que, não abordada como disciplina obrigatória, inerente ao curso de graduação, a formação complementar garante a discussão da oncologia durante a formação em saúde. Neste sentido, destaca-se que $04(36,3 \%)$ artigos mencionaram a formação complementar em oncologia, sendo estes: 02 artigos apresentaram o Curso de Oncologia (Mitchell \& Laing, 2019; Vieira et al., 2016); e 02 estudos discutiram a disciplina eletiva (Guimarães, et al., 2017; Mckillip et al., 2017).

O artigo de Mitchell e Laing (2019) refere o curso em oncologia para os alunos do $4^{\circ}$ ano do curso de graduação em enfermagem, com os seguintes conteúdos: conhecimento fundamental; o papel da enfermagem oncológica; o câncer como doença e seus tratamentos; e cuidado centrado no paciente. O estudo de Vieira et al. (2016) também relata um curso em oncologia, no formato presencial e virtual; e aborda aulas sobre diversos temas relacionados à oncologia. Salienta-se, nessa estratégia de ensino, o uso de plataformas digitais como forma de complementar o ensino em sala de aula. Segundo o estudo, o e-learning, ou ensino virtual, melhora a formação prática dos alunos, aumentando sua responsabilidade no processo de aprendizagem em relação ao método tradicional. Destaca-se a importância de compreender e avaliar novas estratégias de ensino-aprendizagem virtuais, a partir de experiências exitosas voltadas ao ensino em cursos de graduação.

Semelhante aos cursos em oncologia, as disciplinas eletivas também possuem o objetivo de promover a discussão acerca do câncer como problema de saúde pública durante a graduação em saúde. Diferente das disciplinas ofertadas com carga horária relativas aos cursos de graduação, as disciplinas eletivas não são obrigatórias para a formação do profissional. O estudo de Guimarães et al. (2017) relata a oferta de disciplina optativa com carga horária de 30h voltadas aos conteúdos fundamentais da oncologia. Da mesma forma, o estudo de Mckillip et al. (2017) apresenta a disciplina eletiva como estratégia de ensino para a discussão dos principais conteúdos da área em análise.

Destaca-se que, a abordagem em oncologia nos cursos de graduação como formação complementar, devido sua relevância epidemiológica e possibilidade de atuação dos profissionais em todos os níveis da RAS, demonstra o déficit da temática em apreço na matriz curricular obrigatória. Todavia, as estratégias de ensino-aprendizagem por meio de cursos e disciplinas eletivas voltadas à oncologia demonstram alternativas para assegurar a referida discussão, durante a formação dos discentes da área da saúde.

O estudo de Mitchell e Laing (2019) e Hokama et al., (2018), demonstram a estratégia de estudo de caso para o ensino e a aproximação da prática em oncologia. Estudos de casos são estratégias que colaboram para o desenvolvimento da capacidade de observação e inserção do discente como sujeito de seu aprendizado; construtor do conhecimento de forma autônoma; e com a perspectiva da educação para a cidadania, participação na sociedade e compreensão de contextos, a partir de cenários reais da prática (Costa et al., 2018; Moreira, 2015). Dessa forma, é essencial diversificar as estratégias de ensino de modo a estimular o aluno e aproximá-lo da prática, sendo a discussão de estudos de casos eficaz nesse processo de formação discente.

Quanto ao desenvolvimento de seminários, os estudos desenvolvidos por Mitchell e Laing (2019) e Hokama et al., (2018) possuem características em comum e referem que, a partir da discussão dos estudos de caso, os discentes, organizados em grupos, apresentam os resultados em forma de seminário. Mitchell e Laing (2019) refere a divisão dos alunos em pequenos grupos e apresentação do diagnóstico de câncer, o plano de tratamento potencial e medicamentos de apoio. De forma análoga, Hokama et al. (2018) descreve que, a partir de casos motivadores, os estudantes apresentam a relação entre diagnóstico, estadiamento, tratamento e prognóstico. Atenta-se que, a possibilidade de discussão em grupos favorece ao discente o exercício de competências gerenciais, tais como: liderança, comunicação e resolução de conflitos (Caveião et al., 2018). Neste contexto, 
percebe-se que a estratégia de realização de seminários conduzidos pelos próprios discentes, através de propostas estruturadas, voltadas à compreensão da oncologia, é pertinente à inserção discente no seu próprio aprendizado.

No tocante às atividades educativas à população, os artigos de Aragão et al., (2016) e de Freiser et al. (2016) apresentam atividades educativas de extensão voltadas à comunidade. Tais atividades consistem em ações educativas, desenvolvidas em sala de espera de serviços de saúde (Aragão et al., 2016) e estratégia de feira de saúde, voltadas às informações sobre câncer de cabeça e pescoço (Freiser et al, 2016). Entende-se que, as atividades de extensão universitária, através da vivência de experiências educativas, possibilitam troca de experiências entre população e discentes, contribuindo para a formação pessoal e profissional dos discentes (R. Silva et.al. 2020).

Outra estratégia de ensino-aprendizagem em oncologia, a qual dialoga com a extensão universitária, identificada durante a análise dos artigos selecionados foi a Liga Acadêmica, encontrada em 1 artigo (09\%) (Rossato et al., 2020). As Ligas Acadêmicas são organizações estudantis, pautadas no tripé universitário (ensino, pesquisa e extensão), cujas atividades são executadas por meio da colaboração entre docentes, pesquisadores e profissionais do SUS, por iniciativa dos próprios discentes, que se interessam por explorar e aprofundar os conhecimentos sobre um determinado tema (Araújo et al., 2019; Bastos et al., 2012). Tal estratégia indica a preocupação dos discentes com o ensino em oncologia.

Neste contexto, os autores Rossato et al. (2020), apresentam o relato de uma estratégia de discussão de temas relacionados à oncologia, voltados à: percepção e conhecimento dos discentes quanto ao câncer; estigma sobre a doença; e apoio à pessoa com câncer. Essa discussão, fomentada por uma Liga Acadêmica de Oncologia de São Paulo (SP), Brasil, reuniu discentes de graduação para discutir e refletir acerca dos temas propostos, contribuindo para o ensino. O forte impacto das Ligas Acadêmicas no ensino também é exposto no artigo de S. Silva et al. (2020) sinalizado através de demonstrações estatísticas e relatos de participantes.

Percebeu-se por meio da análise dos artigos selecionados que, são diversas as estratégias de ensino empregadas durante a graduação, direcionadas à oncologia. As estratégias descritas dialogam com o ensino teórico e prático quanto à temática em apreço. Todavia, torna-se importante a descrição das estratégias voltadas ao ensino prático em oncologia, por oportunizar aos discentes o encontro com os usuários dos serviços de saúde. Destaca-se que, identificou-se, nos artigos selecionados, o uso de metodologias ativas e estratégias educativas como importantes ferramentas potencializadoras da articulação entre teoria e prática, junto à interação com a comunidade, no que tange à formação para a prática de cuidado em oncologia dos futuros profissionais de saúde.

Categoria 2. Abordagens voltadas aos princípios e diretrizes da Política Nacional de Prevenção e Controle do Câncer na RAS identificadas nas estratégias de ensino.

Esta categoria apresenta as abordagens voltadas aos princípios e diretrizes da Política Nacional de Prevenção e Controle do Câncer na RAS, identificadas nas estratégias de ensino, durante a formação do profissional de saúde para a prática de cuidado em oncologia. Destaca-se que, para o controle do câncer e qualidade de vida da população é necessário um conjunto de ações que permeiam desde a promoção da saúde até os cuidados paliativos, perpassando a vigilância; o monitoramento; e a avaliação das ações de controle do câncer e de seus fatores de risco e proteção. Neste contexto, as estratégias de ensino-aprendizagem devem agregar abordagens que articulem: ações de promoção da saúde e prevenção da doença em apreço, às ações que garantam a proteção e cuidados das pessoas com câncer.

Durante a análise dos artigos selecionados, destacou-se os princípios e as diretrizes da Política Nacional de Prevenção e Controle do Câncer na RAS no ensino em oncologia. Desta forma, 04 (36,3\%) artigos mencionaram a temática de promoção da saúde (Fox, 2020; Rossato et al., 2020; Aragão et al., 2016 e Freiser et al., 2016); 01 (09\%) artigo refere-se à prevenção do câncer (Freiser et al., 2016); 06 (54,5\%) artigos abordaram a fase do diagnóstico da doença (Mitchell \& Laing, 2019; Peñas- 
Felizzola et al., 2017; Aragão et al., 2016; Freiser et al., 2016; Hokama et al., 2018 e Mckillip et al., 2017); 04 (36,3\%) artigos relacionaram-se à fase do tratamento (Mitchell \& Laing, 2019; Arenas et al., 2018; Hokama et al., 2018 e Mckillip Et Al., 2017); e, finalmente, $03(27,2 \%)$ artigos relatam a fase dos cuidados paliativos (Guimarães, et al., 2017; Vieira et al., 2016 e Mckillip et al., 2017). Destaca-se que não se identificou as abordagens de ensino voltadas aos princípios e diretrizes referentes à detecção precoce e às ações voltadas à vigilância; monitoramento; e avaliação das ações de controle do câncer e de seus fatores de risco e proteção, em nenhum artigo selecionado.

Entende-se que, para uma prática de cuidado integral voltada ao controle do câncer na RAS, é preciso que as estratégias de ensino sejam voltadas aos princípios e diretrizes nas ações de prevenção, controle e vigilância do câncer, de modo a preparar o futuro profissional para a prática de cuidado no controle do câncer. Logo, é importante salientar a identificação do menor quantitativo de estudos voltados à formação em saúde nos cursos de graduação com foco na prevenção, detecção precoce e vigilância do câncer, sendo priorizado o momento de formação em saúde articulado aos cuidados após o diagnóstico da doença.

No que se refere à promoção da saúde identificou-se estratégias de ensino voltadas ao encontro com a população, durante a análise do conteúdo dos 04 artigos selecionados (Fox, 2020; Rossato et al., 2020; Aragão et al., 2016 e Freiser et al., 2016). A promoção da saúde é compreendida como atividades voltadas para adoção de práticas sociais e de saúde centradas na equidade, na participação e no controle social (Resolução n. 588, 2018). Neste sentido, a promoção da saúde não restringe a saúde à ausência de doença, mas, que seja capaz de incidir sobre as condições de vida da população (Conselho Nacional de Secretários da Saúde [CONASS], 2016).

Neste sentido, identificou-se nos artigos, atividades educativas voltadas à população relacionadas às atividades de extensão universitária e feiras de saúde. Os autores Rossato et al. (2020), assim como o de Aragão et al. (2016) e Freiser et al. (2016) apresentam a promoção da saúde pautada no encontro e diálogo dos próprios discentes voltada à temática em apreço junto à comunidade acadêmica e à população. No artigo de Fox (2020), a promoção da saúde ocorre através da troca de conhecimentos entre docentes e discentes sobre a oncologia durante a graduação.

O estudo de Rossato et al. (2020) relata a vivência de uma Liga Acadêmica de oncologia na elaboração de estratégias de conversas com discentes de um curso de graduação em enfermagem, sobre os seguintes temas: percepções pessoais sobre a palavra câncer; percepções e conhecimentos sobre o câncer antes e depois da entrada na universidade; estigma em relação ao câncer e apoio à pessoa adoecida por câncer. Trata-se de uma importante estratégia de sensibilização discente, acerca da prática de cuidado em oncologia. No estudo de Aragão et al. (2016), a promoção da saúde, através da educação em saúde voltada à população, apresenta o uso de materiais educativos como brinquedos, bonecos e desenhos para colorir. O estudo discorre sobre a educação em saúde junto a pacientes e acompanhantes em sala de espera, dialogando quanto às dúvidas, exames, procedimentos e o impacto da doença.

Os artigos selecionados, destacam que, ao interagir com a comunidade, é possível criar estratégias de ensino capazes de potencializar a participação e a promoção da saúde da população no seu próprio cuidado. Trata-se de uma abordagem de promoção da saúde para além da relação professor-aluno, envolvendo os discentes em atividades voltadas à população.

Quanto à prevenção do câncer, atenta-se que, é uma abordagem imprescindível, uma vez que a Organização Mundial da Saúde (OMS) considera que cerca de $40 \%$ das mortes por câncer poderiam ser evitadas com ações de prevenção, visto que o câncer é influenciado também por fatores externos (Silva et al., 2020; INCA, 2020). A prevenção do câncer, nos artigos selecionados, foi abordada apenas no estudo de Freiser et al. (2016), através da estratégia de feiras de saúde elaborada por estudantes. Nesta estratégia de ensino-aprendizagem, ocorre a discussão de fatores de risco; sinais e sintomas; e exame físico relacionada ao câncer de cabeça e pescoço. É conhecido que a prevenção do câncer depende de medidas para reduzir ou evitar a exposição aos seus fatores de risco (INCA, 2020). Neste sentido, a prevenção primária do câncer, conceituada pelo INCA como ações com o objetivo de impedir que o câncer se desenvolva, através da adoção de um modo de vida saudável e não exposição 
às substâncias causadoras de câncer (INCA, 2018) é importante durante a graduação, para que o futuro profissional de saúde desenvolva ações de prevenção e, consequentemente, contribuam no controle do câncer.

A análise dos artigos selecionados nesta revisão, ao evidenciar a ausência de artigos específicos quanto às estratégias de ensino que abordassem à deteç̧ão precoce do câncer, possibilitou alertar para a lacuna da produção científica no SUS e na RAS relacionada à essa diretriz. O objetivo da detecção precoce é identificar o câncer quando está localizado no órgão de origem, antes de invadir os tecidos circundantes e órgãos distantes, ou detectar uma lesão pré-cancerosa (World Health Organization [WHO], 2007). Neste sentido, ocorre uma lacuna da produção de conhecimento científico voltado à formação em saúde referente à etapa de detecção precoce do câncer, etapa essencial para o controle da doença em apreço.

Os profissionais de saúde devem ser formados para compreender que o câncer, quando diagnosticado precocemente, tem muito mais probabilidade de responder a um tratamento eficaz (WHO, 2007). Através da análise dos artigos selecionados, identificou-se em 06 artigos selecionados (Mitchell \& Laing, 2019; Peñas-Felizzola et al., 2017; Aragão et al., 2016; Freiser et al., 2016; Hokama et al., 2018 e Mckillip et al., 2017), a apresentação do diagnóstico como abordagem durante o ensino da oncologia nos cursos de graduação em saúde. Assim, infere-se que, a detecção precoce do câncer pode estar associada à formação para o exame diagnóstico, sendo importante ampliar a compreensão das práticas em saúde voltadas à detecção precoce do câncer.

Segundo o "WHO guide for effective programmes", as estratégias necessárias para o diagnóstico do câncer perpassam o aprendizado de sinais e sintomas, assim como a importância de buscar atendimento médico imediato (WHO, 2007). O estudo desenvolvido por Penãs-Felizzola et al. (2017), através da perspectiva dos próprios estudantes, cita o diagnóstico como parte do conteúdo de formação no manejo do paciente oncológico. Da mesma forma, o estudo de Hokama et al. (2018) e Mckillip et al. (2017) apresentam o diagnóstico como parte do conteúdo abordado durante o ensino em apreço. No estudo desenvolvido por Freiser et al. (2016) o diagnóstico é vivenciado pelos discentes, através de exames físicos realizados na população; discussão dos sinais e sintomas; e dos fatores de risco.

O estudo de Silva et al. (2020) corrobora com essa afirmação ao refletir que a forma mais eficaz de controlar a doença é o diagnóstico associado à garantia de um tratamento rápido e adequado. O estudo de Aragão et al. (2016) menciona o diagnóstico referente aos exames e procedimentos ofertados no hospital estudado. Para além de exames diagnósticos, também é abordado a preocupação com o bem estar psicológico do paciente recém diagnosticado e seu acompanhante, através da atenção e escuta comprometidas com o fortalecimento dos sujeitos diante da nova realidade e do tratamento que inicia.

Atenta-se para a discussão do impacto do diagnóstico na vida dos pacientes e familiares, na oferta de uma prática de cuidado pautada na pessoa e não apenas no câncer, como doença. Em estudo sobre o impacto do diagnóstico nas condições socioeconômicas de famílias de crianças e adolescentes com tumores sólidos, é visto que o adoecimento oncológico de um membro do grupo familiar causa impactos em toda a sua estrutura, principalmente ao cuidador da pessoa com câncer (N. Silva, 2020). Desta forma, ofertar apoio à pessoa com câncer e ao cuidador, assim como, o encaminhamento a um serviço de apoio psicológico, são pontos abordados pelo estudo mencionado.

Cabe situar que, para o diagnóstico, a fim de obter melhores prognósticos da doença, se faz necessário a formação de futuros profissionais de saúde voltados aos princípios e diretrizes de promoção, prevenção e detecção precoce do câncer, anteriormente ao tratamento do mesmo; aliado à educação em saúde junto à população, referente aos sinais e sintomas apresentados. Assim, destaca-se que, as estratégias de ensino-aprendizagem voltadas à promoção, prevenção e detecção precoce referentes ao câncer devem ser inerentes à formação para a prática de cuidado em oncologia dos discentes de cursos de graduação na área da saúde.

No que se refere ao tratamento do câncer, a análise evidenciou que os 04 artigos (Mitchell \& Laing, 2019; Arenas et al., 2018; Hokama et al., 2018 e Mckillip et al., 2017) discutem as estratégias de ensino voltadas à abordagem em apreço. O tratamento visa curar doenças, prolongar a vida e melhorar a qualidade de vida remanescente, após o diagnóstico de câncer. Em 
sua maioria, o tratamento eficaz e eficiente está ligado a programas de detecção precoce e a realização de um diagnóstico preciso, feito a partir da história clínica e do exame físico detalhados (INCA, 2020; WHO, 2008). O artigo de Mitchell e Laing (2019), apresenta a abordagem do tratamento através das estratégias de ensino relacionadas à discussão de casos e apresentação de seminários simulando cenários de prática. Dessa forma, pôde-se abordar o tratamento do câncer, durante o referido curso de graduação. Quanto ao estudo desenvolvido pelos autores Arenas et al. (2018) observou-se, durante o ensino da radioterapia para discentes de medicina, as temáticas voltadas para: radioterapia como tratamento; radiobiologia; e o papel da radioterapia em diferentes tipos de câncer.

Atenta-se que, as quatro formas principais de tratamento do câncer: cirurgia, radioterapia, quimioterapia e imunoterapia podem ser usadas em conjunto, alterando quanto à suscetibilidade dos tumores a cada uma das modalidades terapêuticas e à melhor sequência de sua administração (INCA, 2020). O estudo de Mckillip et al. (2017) refere o ensino em saúde voltado ao tratamento do câncer referente às ações de cirurgia, radioterapia e tratamento medicamentoso, como quimioterapia. Tal estratégia corresponde ao preconizado pelo Ministério da Saúde (INCA, 2020) e a OMS (WHO, 2008). O estudo de Hokama et al. (2018) apresenta a proposta dos discentes em montar um plano terapêutico através da identificação do diagnóstico e estadiamento. A estratégia do tratamento, nesse caso, possui uma perspectiva de enfoque no prognóstico do paciente, indo além de apenas diagnóstico e tratamento.

Cabe à discussão, a falta de estudos com abordagem familiar e cuidado centrado na pessoa frente a situação do tratamento. Pacientes com câncer e suas famílias precisam de cuidados centrados na pessoa. $\mathrm{O}$ câncer (e seu tratamento) também tem efeitos emocionais, sociais, psicológicos e consequências espirituais para o paciente e seus familiares (WHO, 2008). Além disso, existem condições financeiras e dificuldades que podem inviabilizar acesso ou adesão ao tratamento oncológico, como a dificuldade de transporte, ausência de recursos financeiros e o não acesso às políticas públicas que respaldam o paciente em tratamento oncológico. Ressalta-se que, mesmo após três meses da confirmação diagnóstica, os usuários não acessam os direitos sociais que são primordiais para garantir melhores condições na realização do tratamento oncológico (N. Silva, 2020).

Atenta-se que, os princípios e diretrizes da Política Nacional para a Prevenção e Controle do Câncer na RAS se encerra nos cuidados paliativos (Portaria n. 874, 2013). Segundo a OMS, cuidados paliativos aprimoram a qualidade de vida dos pacientes e famílias que enfrentam problemas associados com doenças ameaçadoras de vida, através da prevenção e alívio do sofrimento. Utilizam-se meios de identificação precoce; avaliação correta e tratamento da dor; e outros problemas de ordem física, psicossocial e espiritual (INCA, 2020). A qualidade de vida se torna indispensável ao cuidado paliativo, certificando-se do bem-estar do paciente e de seu familiar (Silveira et al., 2020).

A abordagem dos artigos de Vieira et al. (2016) e McKillip et al. (2017) em cuidados paliativos é através da presença dessa temática no currículo de formação do discente de fisioterapia. O artigo de Guimarães et al. (2017) menciona os cuidados paliativos voltados ao câncer pediátrico e salienta o despreparo e insegurança dos discentes de um curso de graduação em enfermagem para lidar com essas situações, principalmente devido à pouca abordagem durante a graduação.

Desta forma, percebeu-se que o foco dos artigos se relaciona às etapas de diagnóstico e tratamento na formação em cursos de graduação em ciências da saúde. Em detrimento de pesquisas referentes à prevenção, detecção precoce, vigilância, monitoramento e avaliação das ações relacionadas ao câncer. Reitera-se a importância do enfoque nas abordagens referentes às estratégias de ensino voltadas à prevenção, por meio do ensino de práticas de cuidados que incidam antes da doença se instalar. Assim como, a abordagem de estratégias de ensino voltadas à compreensão da vigilância do câncer por meio do monitoramento e avaliação das ações de controle, dos fatores de risco e proteção do câncer. Neste sentido, é imprescindível que os discentes possam vivenciar estratégias de ensino voltadas aos princípios e diretrizes da Política Nacional para a Prevenção e Controle do Câncer na RAS. 


\section{Conclusão}

Identificou-se que, as estratégias de ensino para a prática de cuidado em oncologia nos cursos de graduação da área da saúde apresentam-se, nos artigos selecionados, por diversas estratégias de ensino-aprendizagem e abordam os princípios e diretrizes da Política Nacional para a Prevenção e Controle do Câncer na RAS, através das abordagens de: promoção da saúde, prevenção e diagnóstico do câncer; tratamento e cuidados paliativos. Destaca-se que não se identificou estratégias de ensino voltadas à detecção precoce, à vigilância, ao monitoramento e à avaliação das ações relacionadas ao câncer. Tão importante quanto, a identificação das estratégias de ensino desenvolvidas na formação em saúde, a partir da literatura científica, foi a apresentação das referidas estratégias relacionadas à abordagem dos princípios e diretrizes, a partir da referida Política Nacional.

Ao entender a relevância das estratégias de ensino para a prática de cuidado em oncologia nos cursos de graduação na área da saúde, este estudo aponta para as lacunas na produção científica no que tange à formação relacionada às abordagens dos princípios e diretrizes presentes na Política Nacional para a Prevenção e Controle do Câncer na RAS; identifica que o processo de formação do profissional de saúde para o cuidado no controle do câncer tem sido implementado prioritariamente por atividades extracurriculares ou eletivas; e alerta para a necessidade do ensino multiprofissional para o aprendizado em oncologia entre os cursos da saúde. Salienta-se a necessidade de mais estudos na área, com o objetivo de promover a discussão de estratégias de ensino nos cursos de graduação articuladas às abordagens dos princípios e diretrizes da Política Nacional para a Prevenção e Controle do Câncer na RAS.

Desta forma sugere-se a produção de pesquisas científicas na formação em saúde, enfatizando estudos quanto às temáticas relacionadas à oncologia, onde os princípios e diretrizes da Política Nacional para a Prevenção e Controle do Câncer na RAS sejam abordados.

As limitações deste estudo associam-se ao critério de inclusão de artigos disponíveis no formato completo nas bases de dados pesquisadas para leitura e seleção; à seleção de pesquisas desenvolvidas em diferentes países, o que pode representar diferenças curriculares e dificultar a comparação entre as abordagens descritas; e à inclusão de diferentes artigos de diversos cursos de graduação em saúde, com diferentes competências na prática de cuidado no controle do câncer.

\section{Referências}

Aragão, D. A., Uchoa, G. J. B., \& Germano, I. M. P. (2016). Ações na sala de espera de serviço de oncologia: relato de experiência. Revista De Psicologia, 7(1), 242-250. Recuperado de http://www.periodicos.ufc.br/psicologiaufc/article/view/3704

Araujo, C. R. C., Lopes, R. E., Dias, M. S. de A., Ximenes, F. R. G. Neto, Farias, Q. L. T, \& Cavalcante, A. S. P (2019). Contribuição das Ligas Acadêmicas para Formação em Enfermagem. Enfermagem em Foco, 10(6). https://doi.org/10.21675/2357- 707X.2019.v10.n6.2802

Arenas, M., Sabater, S., Biete, A., Lara, P., \& Calvo, F. (2018). Radiation Oncology Teaching Programmes as Part of the Undergraduate Degree in Medicine in Spanish Universities: the Need for an Update of the Contents and Structure. Journal of cancer education: the official journal of the American Association for Cancer Education, 33(2), 352-358. https://doi.org/10.1007/s13187-016-1106-2

Bastos, M. L. S, Trajman A., Teixeira, E. G, Selig, L., \& Belo M. T. C. T. (2012). O papel das ligas acadêmicas na formação profissional. J Bras Pneumol. 38(6):803-5. https://doi.org/10.1590/S1806-37132012000600018

Calil, A. M., \& Prado, C. (2010). Ensino de oncologia na formação do enfermeiro. Revista Brasileira de Enfermagem, 63(4), 671-674. https://dx.doi.org/10.1590/S0034-71672010000400026.

Caveião, C, Peres, A. M., Zagonel, I. P. S., Amestoy, S. C., \& Meier, M. J. (2018). Tendências e estratégias de ensino-aprendizagem utilizadas no desenvolvimento da liderança do enfermeiro. Revista Brasileira de Enfermagem, 71(4), 1531-1539. https://doi.org/10.1590/0034-7167-2017-0455

Conselho Nacional de Secretários da Saúde [CONASS]. (2016). Promoção da Saúde: Propostas do Conselho Nacional de Secretários de Saúde (CONASS) para sua efetivação como política pública no Brasil. https://www.conass.org.br/wp-content/uploads/2016/06/Promoc\%CC\%A7a\%CC\%83o-da-Sau\%CC\%81deCONASS.pdf

Costa, D. A. S., Silva, R. F., Lima, V. V. \& Ribeiro, E. C. O. (2018). Diretrizes curriculares nacionais das profissões da Saúde 2001-2004: análise à luz das teorias de desenvolvimento curricular. Interface - Comunicação, Saúde, Educação, 22(67), 1183-1195. https://dx.doi.org/10.1590/1807-57622017.0376

Fox C. (2020). Why our undergraduate nursing programs need oncology content: Reflections of a nursing instructor. Canadian oncology nursing journal. 30(1), 62-63. https://pubmed.ncbi.nlm.nih.gov/33118982/ 
Freiser, M. E., Desai, D. D., Azcarate, P. M., Szczupak, M., Cohen, E. R., Raffa, F. N., George, J. S., Lo, K., Nayak, C. S., Weed, D. T., \& Sargi, Z. B. (2016) Educational Value of a Medical Student-Led Head and Neck Cancer Screening Event. Official journal of American Academy of Otolaryngology-Head and Neck Surgery, 154(4), 638-644. https://doi.org/10.1177/0194599815626147

Guimarães, T. M. Silva, L. F. da, Santo, F. H. E., Moraes, J. R. M. M. de, Pacheco, S. T. de A. (2017). Cuidado paliativo em oncologia pediátrica na formação do enfermeiro. Revista Gaúcha de Enfermagem, 38(1), e65409. https://doi.org/10.1590/1983-1447.2017.01.65409

Gonçalves, M. F., Gonçalves, A. M., \& Gonçalves, I. M. F. (2020). Aprendizagem baseada em problemas: uma abordagem no ensino superior na área da saúde. Práticas Educativas, Memórias E Oralidades - Rev. Pemo, 2(1), 1-12. https://doi.org/10.47149/pemo.v2i1.3676

Hokama, P. O. M., Hokama, N. K. \& Batista, N. (2018). Caso Motivador como Estratégia Problematizadora e Integradora no Ensino Médico em um Curso de Oncologia. Revista Brasileira de Educação Médica, 42(4), 165-174. https://doi.org/10.1590/1981-52712015v42n4rb20170080

Instituto Nacional De Câncer José Alencar Gomes Da Silva. (2020). ABC do câncer: abordagens básicas para o controle do câncer (2a ed.). Rio de Janeiro, RJ: INCA. https://www.inca.gov.br/publicacoes/livros/abc-do-cancer-abordagens-basicas-para-o-controle-do-cancer

Instituto Nacional De Câncer José Alencar Gomes Da Silva. (2019). Estimativa 2020: incidência de câncer no Brasil. Rio de Janeiro, RJ: INCA. https://www.inca.gov.br/publicacoes/livros/estimativa-2020-incidencia-de-cancer-no-brasil

Instituto Nacional De Câncer José Alencar Gomes Da Silva. (2018). Prevenção e fatores de risco. Rio de Janeiro. Recuperado em 02 abr 2020 de: https://www.inca.gov.br/causas-e-prevencao/prevencao-e-fatores-de-risco

Lins, F., \& Souza, S. (2018). Formação dos enfermeiros para o cuidado em oncologia. Revista de Enfermagem UFPE on line, 12(1), 66-74. https://doi.org/10.5205/1981-8963-v12i1a22652p66-74-2018

Moreira, A. E. C. (2015). O Papel docente na seleção das Estratégias de Ensino. Anais da XVI Semana da Educação e o VI Simpósio de Pesquisa e PósGraduação em Educação: "Desafios atuais para a educação". Londrina, Brasil. https://psicod.org/o-papel-docente-na-seleco-das-estratgias-de-ensino.html

McKillip, R. P., Hahn, O. M., Bartkowiak, B., Rosenberg, C. A., Olopade, O. I., Arora, V. M., \& Golden, D. W. (2019). Implementation of a Novel Medical School Multidisciplinary and Interprofessional Oncology Curriculum: a Mixed Method Study. Journal of cancer education: the official journal of the American Association for Cancer Education, 34(1), 50-55. https://doi.org/10.1007/s13187-017-1264-x

Mitchell, C., \& Laing, C. M. (2019). Revision of an undergraduate nursing oncology course using the Taylor Curriculum Review Process. Canadian oncology nursing journal, 29(1), 47-51. https://doi.org/10.5737/236880762914751

Nalom, D. M. F., Ghezzi, J. F. S. A., Higa, E. F. R., Peres, C. R. F. B., \& Marin, M. J. S. (2019). Ensino em saúde: aprendizagem a partir da prática profissional. Ciência \& Saúde Coletiva, 24(5), 1699-1708. https://doi.org/10.1590/1413-81232018245.04412019

Oxford Centre for Evidence-based Medicine. (2009). Levels of Evidence. https://www.cebm.ox.ac.uk/resources/levels-of-evidence/oxford-centre-for-evidencebased-medicine-levels-of-evidence-march-2009

Peñas-Felizzola, Olga L., Parra-Esquivel, Eliana I., \& Gómez-Galindo, Ana M. (2018). Terapia ocupacional en oncología: experiencias en prácticas académicas y revisión de literatura. Revista de Salud Pública, 20(1), 45-52. https://doi.org/10.15446/rsap.v20n1.62227

Portaria n. 874 de 16 de maio de 2013 (2013). Institui a Política Nacional para a Prevenção e Controle do Câncer na Rede de Atenção à Saúde das Pessoas com Doenças Crônicas no âmbito do Sistema Único de Saúde (SUS). 2013. http://bvsms.saude.gov.br/bvs/saudelegis/gm/2013/prt0874_16_05_2013.html

Resolução CNE/CES no. 3, de 07 de novembro de 2001. (2001). Institui as Diretrizes Curriculares Nacionais do Curso de Graduação em Enfermagem. 2001. http://portal.mec.gov.br/cne/arquivos/pdf/CES03.pdf

Resolução $\mathrm{N}^{\circ}$ 588, de 12 de Julho de 2018. (2018). Instituí a Política Nacional de Vigilância em Saúde (PNVS). http://conselho.saude.gov.br/resolucoes/2018/Reso588.pdf

Rossato L, Panobianco M. S., Scorsolini-Comin F. (2020) Grupo operativo com estudantes de enfermagem: vivência em uma Liga Acadêmica de Oncologia. Rev baiana enferm. 34:e34690. https://doi.org/10.18471/rbe.v34.34690

Silva, C. A., Cavalcante, G., Ramos, L., Anfe, M., Castro, M., Mello, T., Silva, R., \& Bonini, L. (2019). Humanização No Ensino De Graduação No Curso De Medicina. Diálogos Interdisciplinares, 8(10), 118-132. https://doi.org/10.1590/1981-52712015v39n3e00092015

Silva, G. A. e, Moura, L. de, Curado, M. P., Gomes, F., Otero, U., Rezende, L. F., Daumas, R. P., Guimarães, R. M., Meira, K. C., Leite, I., Valente, J. G., Moreira, R. I., Koifman, R., Malta, D. C., Mello, M. S., Guedes, T. W., \& Boffetta, P. (2016). The Fraction of Cancer Attributable to Ways of Life, Infections, Occupation, and Environmental Agents in Brazil in 2020. PloS one, 11(2), e0148761. https://doi.org/10.1371/journal.pone.0148761

Silva, N. C. F. da, Hora, S. S da, \& Lima, F. F da S. (2020). O Impacto do Diagnóstico nas Condições Socioeconômicas das Famílias de Crianças e Adolescentes com Tumores Sólidos. Revista Brasileira De Cancerologia, 66(3), e-131104. https://doi.org/10.32635/2176-9745.RBC.2020v66n3.1104

Silva, R. K.S, Carvalho, D.S, Silva E. G., Pereira A. R. S, Martins J. A., \& Júnior, I. G. C. (2020). Extensão universitária como um caminho para transcender o ensino de oncologia. Braz. J. of Develop.,Curitiba, 6(8), 56350-56361. https://doi.org/10.34117/bjdv6n8-157

Silva S. S. F da, Cavalcante C. B. T. L., Anizio, M. de S., Nunes, B. L. R., Pinto, A. C. S., \& Paula, D.G. de. Profile and production of academic health science leagues in Brazil: an integrative review. RSD. 9(9):743997775. https://rsdjournal.org/index.php/rsd/article/view/7775

Silveira, P. J. da, Costa, A. E. K. da, Lohmann, P. M., \& Lavall, E. (2020). Integrative review: palliative care in oncological patients. Research, Society and Development, 9(2), e144922136. https://doi.org/10.33448/rsd-v9i2.2136

Souza, M. T., Silva, M. D., \& Carvalho, R. (2010). Revisão integrativa: o que é e como fazer. Einstein. 8(1), 102-106. https://doi.org/10.1590/s167945082010rw1134 
Research, Society and Development, v. 10, n. 5, e43310515080, 2021

(CC BY 4.0) | ISSN 2525-3409 | DOI: http://dx.doi.org/10.33448/rsd-v10i5.15080

Sung, H., Ferlay, J., Siegel, R. L., Laversanne, M., Soerjomataram, I., Jemal, A., \& Bray, F. (2021). Global cancer statistics 2020: GLOBOCAN estimates of incidence and mortality worldwide for 36 cancers in 185 countries. CA: a cancer journal for clinicians. https://doi.org/10.3322/caac.21660

Vieira, R. A. da C., Lopes, A. H., Sarri, A. J., Benedetti, Z. C., \& de Oliveira, C. Z. (2017). Oncology E-Learning for Undergraduate. A Prospective Randomized Controlled Trial. Journal of cancer education: the official journal of the American Association for Cancer Education, 32(2), 344-351. https://doi.org/10.1007/s13187-015-0979-9

World Health Organization. (2007). Cancer control: knowledge into action: WHO guide for effective programmes (Module 3). Switzerland. https://apps. who.int/iris/bitstream/handle/10665/43743/9241547338_eng.pdf,jsessionid=499FB5A0D3BE62AFCA5F990E4911580E?sequence=1

World Health Organization. (2008). Cancer control: knowledge into action: WHO guide for effective programmes (Module 4). Switzerland. https://apps.who.int/iris/bitstream/handle/10665/43827/9789241547406_eng.pdf?sequence=1 